\title{
MCAT revisions finalized
}

A cademic administrators will substantially reshape the Medical College Admission Test (MCAT) commencing in 2015 as part of a bid to produce more wellrounded physicians.

The Association of American Medical Colleges recently approved final revisions to the exam following a comprehensive review that began in the fall of 2008. Introduced in 1928, the MCAT is annually written by about 10000 medical school applicants in Canada. It was last revised in 1991 and currently consists of four subtests: biological sciences, physical sciences, verbal reasoning and a writing sample. The aim of the fifth review was to retain its value in thinning the field, while improving its predictive capacity to assess which candidates might ultimately make the best medical practitioners (www.cmaj .ca/lookup/doi/10.1503/cmaj.109-3903).

"Being a good physician is about more than scientific knowledge," Dr. Darrell Kirch, president and CEO of the Association of American Medical Colleges, wrote in an open letter to pre-med students regarding the new version of the MCAT (www.aamc.org /newsroom/reporter/march2012/276772 /word.html).

Arguing that the future will require "a different kind of physician," who is more "culturally competent," Kirch added that "it is about understanding people - how they think, interact, and make decisions. Together with a solid foundation in the natural sciences, an understanding of behavior, perception, culture, poverty, and other concepts from psychology and sociology all contribute to the well-rounded physician."

"Of course, we want our doctors to understand how chemical compounds interact so our prescriptions do not have adverse interactions. But we also want our doctors to have good bedside manner, communication skills, and an ability to interact with people. After nearly 60 years, I still remember my pediatrician, Dr. Bramley — not for his

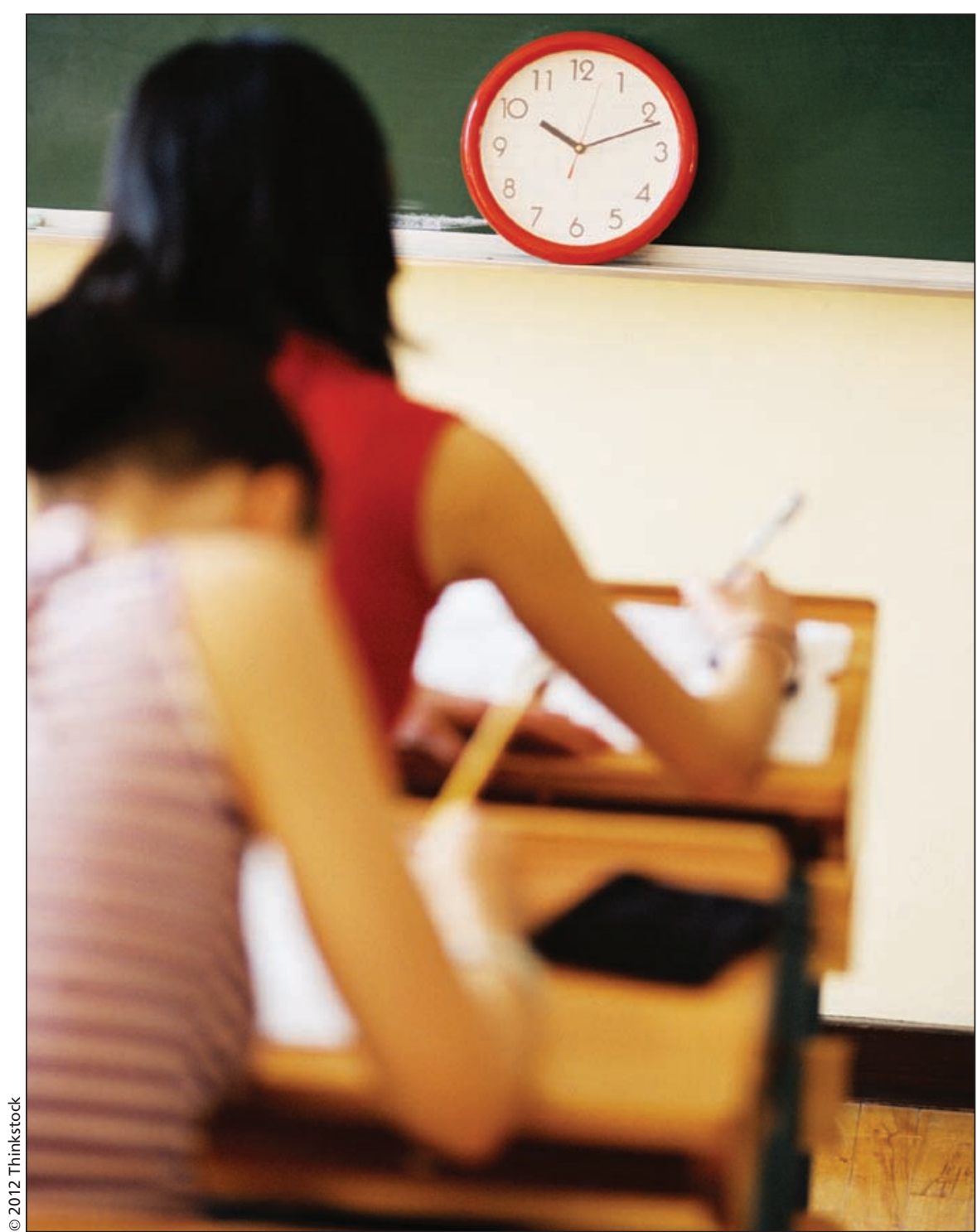

The revised Medical College Admission Test is projected to take 6.5 hours to complete rather than 4.5 hours.

class rank or MCAT exam score, but for his kindness, compassion, and how much he truly cared."

Medical schools in Canada that require applicants to take the MCAT will automatically be adopting the new iteration of the test, said Geneviève Moineau, vice president of education for the Association of Faculties of Medicine of Canada.

Advocates of a revised MCAT had contended changes were needed to allow applicants from a broader undergraduate spectrum an opportunity to pursue a medical career and might somehow also help to redress concerns about a growing demographic imbalance in the medical student population, which is increasingly the domain of those from wealthier families (www .cmaj.ca/lookup/doi/10.1503/cmaj.0614 77). Some schools, such as the McGill University School of Medicine in Montréal, Quebec, have even dispatched the 
MCAT to the dustbins of history as they aim to diversity the composition of incoming classes (www.cmaj.ca/lookup /doi/10.1503/cmaj.109-3679).

The fifth iteration of the MCAT will create a subtest on behavioural and social sciences principles, including biological concepts related to behaviour and mental processes. It will also eliminate the writing sample, which was found to be of little value in terms of predicting an individual's academic success in medical school. Elimination of the writing sample will occur even earlier than 2015. Commencing next January, it will be replaced by an unscored trial section consisting of content from one of the subject areas to be introduced in the new version of the test.

Also to be scrapped is the verbal reasoning section, which will be reshaped into a "critical analysis and reasoning skills" component that will involve analyzing and evaluating passages from disciplines in the realms of social sci- ence and the humanities, such as history, anthropology, music and literature.

As well, the test's estimated completion time has been boosted to 6.5 hours from 4.5 hours. The revisions were based on recommendations made by a 21-member advisory committee appointed by the American Association of Medical Colleges in 2008 (www .aamc.org/download/273766/data/final mr5recommendations.pdf).

The need for physicians who are more familiar with the social and behavioural sciences is the product of growing awareness in North America "of the behavioural and socio-cultural determinants of health, the ways in which behaviour plays a critical role in the health and health outcomes in our population, and the ways in which differential access to resources actually plays a role in differences in health and health outcomes," says Karen Mitchell, senior director of admissions testing for the Association of American Medical Colleges.
The changes should also result in an exam with greater predictive validity regarding likely success in medical school, Mitchell adds. "The blueprints for the exam were based on a great deal of data that describe what medical school faculty said they would like students to know when they walk in the door."

In crafting the new MCAT, the advisory committee consulted with a number of blue-ribbon panels on the preferred competencies of students, organized more than 90 outreach events and surveyed about 2700 medical administrators, students and residents. "All of that data kind of pointed in the same direction," Mitchell says. "They all suggested that we should be testing the things the new exam will test because that's what the medical school folks said students needed to know in order to succeed in medical school." - Michael Monette, CMAJ and Chris Hemond, Ottawa, Ont.

CMAJ 2012. DOI:10.1503/cmaj.109-4198 